\title{
Nuevas observaciones sobre la fenología y distribución espacial de los artrópodos en el dosel y sotobosque de un rodal mixto mediterráneo (Collserola, Cataluña), con énfasis en Diptera e Hymenoptera
}

\section{Jorge Mederos}

Museu de Ciències Naturals de Barcelona. Departament d'Artròpodes

Passeig Picasso, s/n. 08003 Barcelona

mederos@gmail.com

Aneta Kuncová

Joan Sala-Coromina

Juli Pujade-Villar

Universitat de Barcelona. Facultat de Biologia

Av. Diagonal, 645. 08028 Barcelona

tanea21@gmail.com

jsalacor@gmail.com

jpujade@ub.edu

\section{Resumen}

El presente trabajo es una continuación del monitoreo permanente que se viene realizando desde 2009 en el Parc Natural de la Serra de Collserola, y se centra en la actividad de las familias de Diptera e Hymenoptera muestreadas en un bosque mixto mediterráneo PinusQuercus. De abril a octubre de 2012 se realizaron muestreos en el dosel y sotobosque, con un total de 7789 especímenes muestreados y resultando Diptera $(1393 ; 17,9 \%)$ e Hymenoptera $(1039 ; 13,3 \%)$ entre los grupos dominantes en el área. Se encontró una clara estratificación de la fauna, donde el dosel se mostró no solo como el estrato donde se desarrolla el mayor volumen de la actividad de los artrópodos, sino también como el estrato más biodiverso. Estos resultados contrastan con estudios realizados anteriormente en la misma área, donde el sotobosque jugaba un papel dominante sobre el dosel. Las diferencias entre los resultados del primer estudio y los obtenidos en este trabajo evidencian la necesidad de focalizar la atención hacia los diversos factores que marcan la actividad de los artrópodos entre estratos con exposición solar total y parcial.

Palabras clave: artrópodos; bosque mediterráneo; dosel; sotobosque; fenología; Diptera; Hymenoptera; Cataluña. 


\begin{abstract}
Further observations on the phenology and spatial distribution of arthropods in the canopy and understory of a Mediterranean mixed stand (Collserola, Catalonia), with emphasis on Diptera and Hymenoptera
\end{abstract}

This work is part of permanent monitoring that has been conducted since 2009 in the Serra de Collserola Natural Park, and focuses on the activities of families of Diptera and Hymenoptera sampled in a Mediterranean mixed forest of Pinus-Quercus. From April to October of 2012, samples were taken in the canopy and understory, with a total of 7789 specimens with Diptera $(1393 ; 17.9 \%)$ and Hymenoptera $(1039 ; 13.3 \%)$ among the dominant groups. We found a clear stratification of arthropods, where the canopy showed not only as the layer where the bulk of the activity takes place, but also as the most biodiverse stratum. These results contrast with previous studies in the same area where the understory played a dominant role compared to the canopy. The differences between the results of the first study and those obtained in this study show the need to focus attention on the various factors that influence the activity of arthropods between strata with full and partial sun exposure.

Keywords: arthropods; Mediterranean forest; canopy; understory; phenology; Diptera; Hymenoptera; Catalonia.

\title{
Introducción
}

El presente estudio forma parte de un proyecto a largo plazo iniciado en 2009 en el Parc Natural de la Serra de Collserola, un espacio natural de más de 8000 ha dentro de la Cordillera Litoral Catalana y al norte de la ciudad de Barcelona. La importancia de este parque reside en su función como refugio para una destacable biodiversidad, actuando además como corredor intermedio entre otros parques naturales cercanos. Además de encontrarse rodeado de numerosas infraestructuras de transporte (carreteras, autopistas, vías ferroviarias), la presencia humana en el interior de la sierra es elevada e histórica, a lo cual se ha de sumar el incremento con los años del uso lúdico de este espacio. Todos estos factores pueden incidir más o menos negativamente en la salud de los ecosistemas presentes en esta área natural, por lo que el órgano gestor del parque ha incluido entre sus prioridades promover iniciativas para aumentar el conocimiento sobre la biodiversidad presente en Collserola. La base de la biodiversidad del parque se debe sin duda a la diversidad de su paisaje. Aunque dominado por un ambiente eminentemente forestal, en el parque también están presentes distintas unidades de paisaje típicas del mosaico mediterráneo. Pero son los ambientes de bosques mixtos de pinares y encinares Pinus-Quercus, así como los encinares con robles, los que predominan.

Este estudio es una nueva aportación sobre la fenología y ecología de los dípteros e himenópteros en el área de estudio, y que sirve de complemento a una serie de trabajos publicados con anterioridad sobre la fauna de artrópodos presentes tanto en el dosel como en el sotobosque de esta misma área (Mederos-López \& Pujade-Villar, 2011; Mederos-López et al., 2012; Mederos-López \& Baz-Ramos, 2012; Bentanachs et al., 2012; Viñolas 2011a, b; Viñolas et al., 2012a, b; Viñolas 2013a, b, c, d; Viñolas \& Masó, 2013). En esta ocasión, se retoma y 
discute la composición y fenología anual de las diversas familias de Diptera e Hymenoptera presentes en esta localidad, la presencia/ausencia de esta fauna en los dos estratos estudiados (dosel y sotobosque) y se ofrecen diversos apuntes sobre la ecología de algunas especies o grupos de interés.

\section{Materiales y métodos}

Para una descripción detallada del área de estudio y características climáticas puede consultarse los trabajos de Mederos-López \& Pujade-Villar (2011) y Mederos-López et al. (2012). Los muestreos se realizaron de abril a octubre de 2012, en la zona conocida como Can Balasc (P. N. de la Serra de Collserola), empleándose dos trampas Malaise de interceptación (SLAM Trap II - Standard MegaView Science Co., Ltd.), una en el sotobosque a un metro del suelo y otra a ocho metros de altura sobre el dosel superior con dominancia de Quercus ilex y Q. cerrioides y sin la interferencia de un dosel de Pinus halepensis, por lo que la exposición a la radiación solar es total. Ambas trampas fueron ubicadas en la misma vertical (Fig. 1).

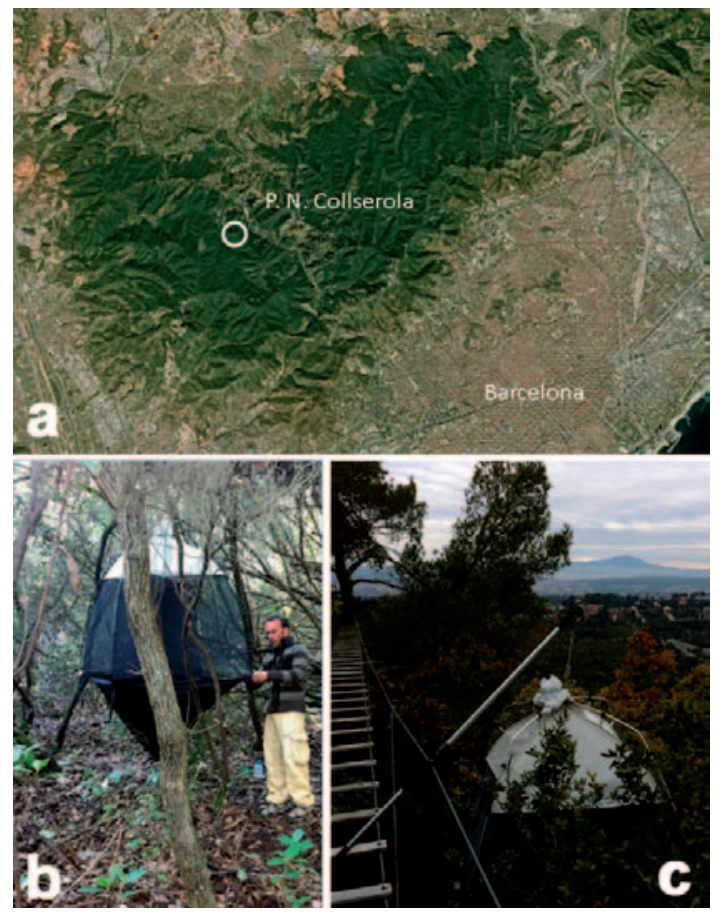

Figura 1. (a) Parc Natural de la Serra de Collserola, al norte de la ciudad de Barcelona y localización del área de estudio en el Turó de Can Balasc. Trampas Malaise de intercepción utilizadas para los muestreos, ubicadas en la misma vertical en el sotobosque (b) y dosel (c). 
El acceso al dosel superior se realizó mediante técnicas de progresión por cuerdas y una pasarela ubicada sobre el dosel, técnicas comúnmente utilizadas en las prospecciones de este estrato (Moffett \& Lowman, 1995; Mitchell et al., 2002). La metodología de muestreos utilizada fue la captura ininterrumpida de material durante el tiempo de estudio, sin el empleo de ningún tipo de atrayente, feromonas o luz. Las muestras fueron retiradas mensualmente. En ambas trampas se usó etanol al 70\%, tanto para la captura del material como para la conservación. No se excluyó ningún espécimen capturado de ningún grupo ni talla. Para la separación e identificación de las muestras por familias de Diptera se siguió la guía de identificación para Europa (Oosterbroek, 2006). Para la separación e identificación de Hymenoptera se siguió el criterio de Pujade-Villar \& Fernández-Gayubo (2004). Para el cálculo de la biodiversidad por estratos se utilizó el índice de Shannon-Wiener. El material colectado se encuentra depositado en la colección privada del autor [JML].

\section{Resultados}

Con un total de 7789 especímenes muestreados durante el estudio (de abril a octubre de 2012), la abundancia de artrópodos fue en general mucho mayor en el dosel que en el sotobosque, con 5499 y 2290 individuos respectivamente. El número de órdenes capturados en el dosel fue de 13 mientras 15 lo fueron en el sotobosque, siendo Microcoryphia y Pseudoscorpiones los dos órdenes muestreados exclusivamente en el sotobosque. En el dosel, los órdenes más abundantes resultaron Hemiptera, Díptera, Lepidoptera e Hymenoptera, mientras que en el sotobosque fueron Collembola, Hemiptera, Psocoptera e Hymenoptera (Fig. 2). Aunque la mayor parte de órdenes se encuentran en ambos estratos, la distribución de sus abundancias no es la misma. Se observa como los cuatro órdenes más abundantes representan un $80,4 \%$ de la muestra general capturada en el dosel mientras que en el sotobosque solo representan el 37,5\% del total. Por otro lado, el sotobosque resulta ser un estrato más biodiverso que el dosel (índice de Shannon-Wiener (H): 3.29 y 2.69 respectivamente).

Los órdenes que muestran una mayor diferencia entre estratos son principalmente los voladores. Lepidoptera, Diptera, Hemiptera, Hymenoptera y Coleoptera se muestran con mayor proporción en el dosel, mientras que los órdenes Psocoptera y Blattodea se colectaron con mayor abundancia en el sotobosque. Por otro lado, como era de esperar, los órdenes no voladores (Acari, Araneae, Microcoryphia y Collembola, por ejemplo) fueron colectados mayoritariamente en el sotobosque.

Observando la fenología anual de la totalidad de artrópodos del dosel y sotobosque, se aprecian dos máximos de actividad, uno en mayo y otro en agosto. Los altos valores de precipitación registrados durante abril (Fig. 3), con el establecimiento de la primavera y la abundancia de recursos tróficos como la floración, podrían explicar esta marcada actividad de artrópodos. Sin embargo, el máximo de agosto podría estar influenciado por la temperatura media alcanzada durante ese mes, la máxima de todo el año. 


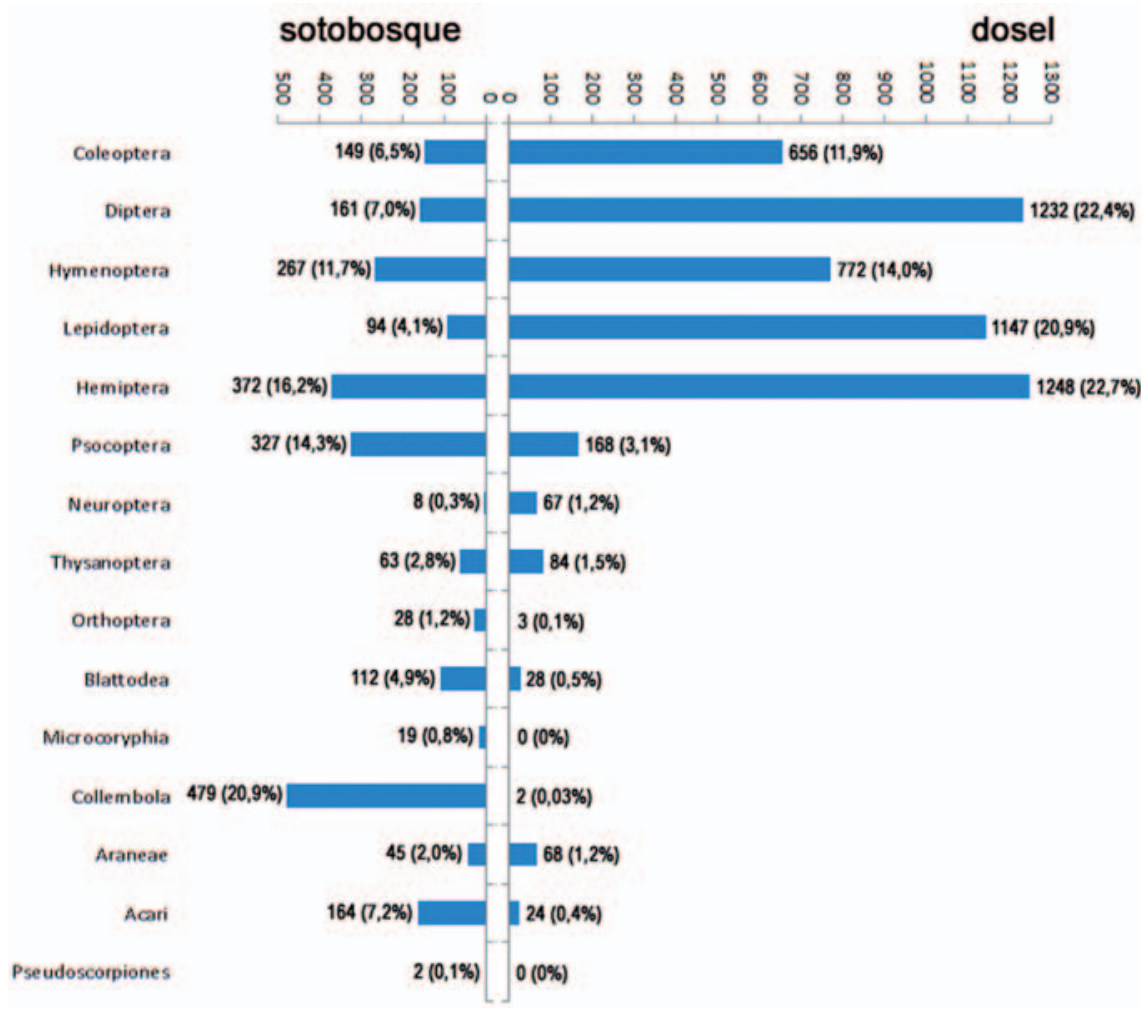

Figura 2. Número de especímenes y abundancia relativa (\%) de los órdenes capturados en los dos estratos estudiados.
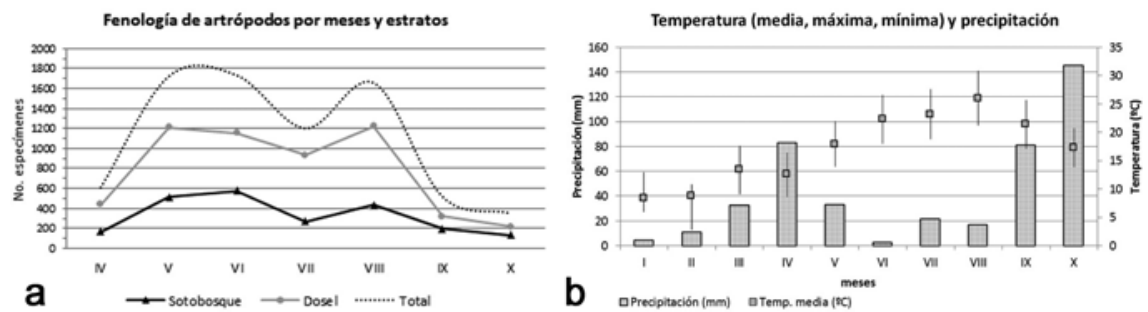

Figura 3. (a) Fenología del total de artrópodos durante el periodo estudiado (de abril a octubre de 2012) y total de especímenes muestreados por estratos. (b) Precipitación (barras) y temperatura media (puntos) en el área de estudio (datos meteorológicos: Observatori Fabra). 
Si se toma la actividad de cada estrato por separado a lo largo del período estudiado, se aprecia una sincronía en cuanto a los máximos de actividad pero una marcada diferencia en cuanto a la abundancia. Además, en el dosel se registran oscilaciones muy marcadas de la abundancia, mientras en el sotobosque la situación es más estable.

Del total de artrópodos capturados en ambos estratos, 1393 correspondieron a Diptera $(17,9 \%)$ y este valor lo ubica solo por detrás de Hemiptera, de los que fueron capturados 1620 especímenes (20,8\%), seguidos por Lepidoptera $(1241 / 15,9 \%)$, Hymenoptera $(1039 / 13,3 \%)$ y Coleoptera $(805 / 10,3 \%)$. Hymenoptera y Hemiptera presentaron un pico de abundancia a finales de primavera, Lepidoptera en verano y Diptera presentó dos picos de abundancia (Fig. 4).

En el caso particular de Diptera, se capturaron un total de treinta familias durante todo el estudio (Tabla 1), siendo más abundantes los Nematocera al aportar al conjunto de este orden el 85,7\% (1194). Con 706 especímenes Cecidomyiidae resultó la familia más abundante (Fig. 5), aportando el 50,7\% del total de Diptera, un resultado que se repite en estudios anteriores en esta misma área (Mederos-López \& Pujade-Villar, 2011), y seguida por Chironomidae (176/12,6\%), Ceratopogonidae $(124 / 8,9 \%)$ y Sciaridae $(92 / 6,6 \%)$. Octubre resultó el mes con menor diversidad, con solo 8 familias capturadas mientras mayo y junio ofrecieron el máximo, con 17 familias. En cuanto a la actividad, el máximo de todo el período se alcanzó en mayo, con 353 especímenes, correspondiendo a Cecidomyiidae el 73,4\% del total. La menor actividad se registró en septiembre con 90 especímenes, aportando Cecidomyiidae el 41,1\%.

En cuanto a los diferentes estratos estudiados, el dosel aportó el $88 \%$ del total de dípteros muestreados durante todo el período, con 1232 especímenes (Tabla 1, Fig. 6). Este valor muestra una situación muy diferente a la observada con ante-

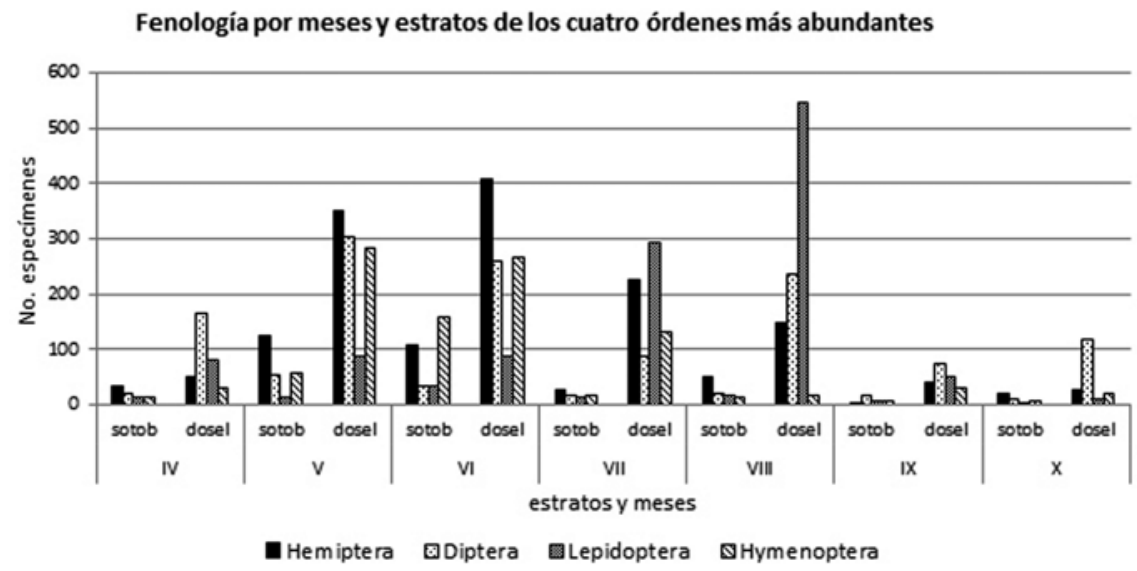

Figura 4. Fenología y actividad por estratos de los cuatro órdenes de artrópodos más abundantes durante el período de estudio. 

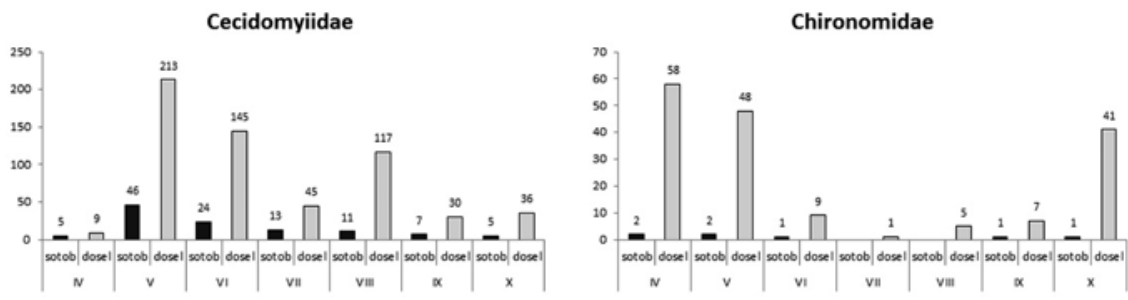

Ceratopogonidae

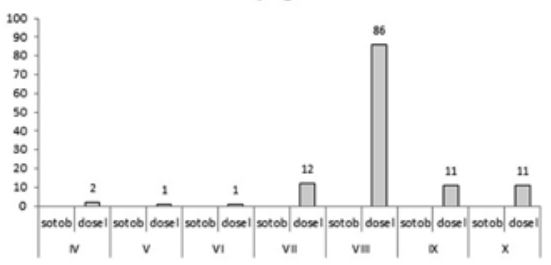

Sciaridae

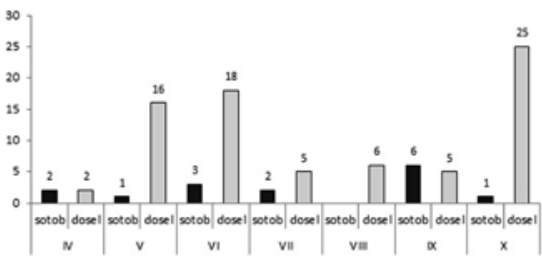

Bibionidae
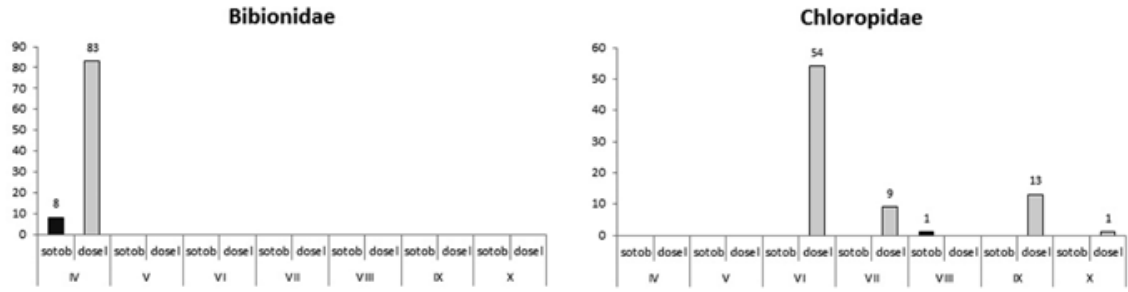

Figura 5. Fenología y actividad por estratos de las familias de Diptera más abundantes muestreadas durante el período de estudio.

rioridad en esta misma área (Mederos-López \& Pujade-Villar, 2011; MederosLópez et al., 2012), y cuyas probables causas se discuten más adelante. En total (Tabla 1), 27 familias fueron capturadas en el dosel, de las cuales 15 se encontraron exclusivamente en este estrato, mientras que en el sotobosque fueron capturadas 15 familias (3 exclusivamente en este estrato).

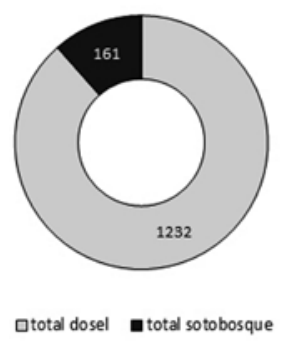

\section{Total Diptera}

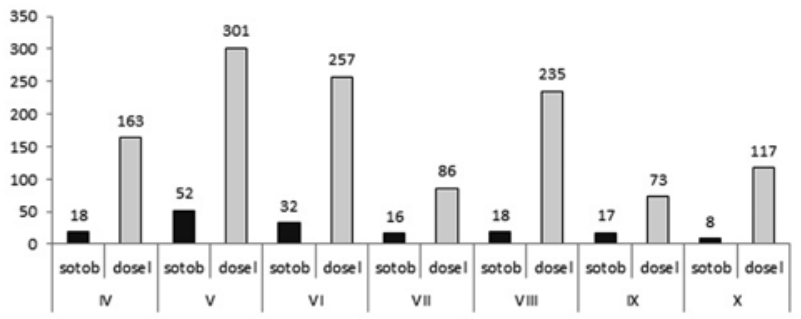

Figura 6. Total de especímenes de Diptera capturados por estratos durante el estudio, de abril a octubre de 2012, y aporte por estrato y mes. 
Tabla 1. Familias y número de especímenes de Diptera muestreados por estratos de abril a octubre de 2012: (*) familias capturadas exclusivamente en el sotobosque; (**) familias capturadas exclusivamente en dosel

\begin{tabular}{|c|c|c|c|c|c|}
\hline \multicolumn{2}{|c|}{ DIPTERA } & \multicolumn{2}{|c|}{ IV } & \multicolumn{2}{|c|}{$\mathbf{V}$} \\
\hline Superfamilia & Familia & dosel & sotob & dosel & sotob \\
\hline BIBIONOIDEA & Bibionidae & 83 & 8 & & \\
\hline \multirow[t]{4}{*}{ SCIAROIDEA } & Mycetophilidae** & & & 1 & \\
\hline & Keroplatidae* & & & & 1 \\
\hline & Sciaridae & 2 & 2 & 16 & 1 \\
\hline & Cecidomyiidae & 9 & 5 & 213 & 46 \\
\hline PSYCHODOIDEA & Psychodidae** & & & 1 & \\
\hline \multirow[t]{3}{*}{ CHIRONOMOIDEA } & Simuliidae ${ }^{* *}$ & & & & \\
\hline & Ceratopogonidae** & 2 & & 1 & \\
\hline & Chironomidae & 58 & 2 & 48 & 2 \\
\hline STRATIOMYOIDEA & Stratiomyidae & & & & \\
\hline ASILOIDEA & Asilidae & & & & \\
\hline \multirow[t]{2}{*}{ EMPIDOIDEA } & Hybotidae** & & & & \\
\hline & Dolichopodidae & & & 4 & \\
\hline PLATYPEZOIDEA & Phoridae & & & 3 & \\
\hline \multirow[t]{2}{*}{ SYRPHOIDEA } & Syrphidae** & & & & \\
\hline & Pipunculidae** & 1 & & 2 & \\
\hline TEPHRITOIDEA & Tephritidae** & & & & \\
\hline \multirow[t]{2}{*}{ LAUXANIOIDEA } & Lauxaniidae* & & & & \\
\hline & Chamaemyiidae** & 1 & & & \\
\hline SCIOMYZOIDEA & Sciomyzidae** & & & 1 & \\
\hline OPOMYZOIDEA & Agromyzidae** & & & 2 & \\
\hline \multirow[t]{2}{*}{ CARNOIDEA } & Cryptochetidae** & & & & \\
\hline & Chloropidae & & & & \\
\hline SPHAEROCEROIDEA & Trixoscelididae** & & & 1 & \\
\hline EPHYDROIDEA & Drosophilidae* & & & & \\
\hline \multirow[t]{2}{*}{ MUSCOIDEA } & Anthomyiidae & 1 & & 4 & 1 \\
\hline & Muscidae & 1 & & & 1 \\
\hline \multirow[t]{3}{*}{ OESTROIDEA } & Calliphoridae & 5 & 1 & 1 & \\
\hline & Sarcophagidae** & & & & \\
\hline & Tachinidae** & & & 3 & \\
\hline TOTAL & & 163 & 18 & 301 & 52 \\
\hline
\end{tabular}




\begin{tabular}{|c|c|c|c|c|c|c|c|c|c|c|}
\hline \multicolumn{2}{|c|}{ VI } & \multicolumn{2}{|c|}{ VII } & \multicolumn{2}{|c|}{ VIII } & \multicolumn{2}{|c|}{ IX } & \multicolumn{2}{|c|}{$\mathbf{X}$} & \multirow[b]{2}{*}{ TOTAI } \\
\hline dosel & sotob & dosel & sotob & dosel & sotob & dosel & sotob & dosel & sotob & \\
\hline & & & & & & & & & & 91 \\
\hline & & & & & & & & & & 1 \\
\hline & & & & & & & & & & 1 \\
\hline 18 & 3 & 5 & 2 & 6 & & 5 & 6 & 25 & 1 & 92 \\
\hline \multirow[t]{3}{*}{145} & 24 & 45 & 13 & 117 & 11 & 30 & 7 & 36 & 5 & 706 \\
\hline & & & & 1 & & & & & & 2 \\
\hline & & & & 1 & & & & & & 1 \\
\hline 1 & & 12 & & 86 & & 11 & & 11 & & 124 \\
\hline 9 & 1 & 1 & & 5 & & 7 & 1 & 41 & 1 & 176 \\
\hline 1 & 1 & 2 & & & & 1 & & & & 5 \\
\hline 1 & & 2 & & & 1 & & & & & 4 \\
\hline 3 & & 1 & & & & 1 & & & & 5 \\
\hline 3 & 1 & & & & 2 & 1 & 2 & & & 13 \\
\hline 11 & 1 & 2 & & 6 & & 1 & & & & 24 \\
\hline 1 & & & & & & & & & & 1 \\
\hline \multirow[t]{5}{*}{2} & & 4 & & 8 & & & & & & 17 \\
\hline & & & & 1 & & & & & & 1 \\
\hline & 1 & & & & 3 & & 1 & & & 5 \\
\hline & & & & & & & & & & 1 \\
\hline & & & & & & & & & & 1 \\
\hline \multirow[t]{2}{*}{1} & & & & & & & & 1 & & 4 \\
\hline & & 1 & & 2 & & 1 & & & & 4 \\
\hline 54 & & 9 & & & 1 & 13 & & 1 & & 78 \\
\hline \multirow[t]{2}{*}{1} & & & & & & & & & & 2 \\
\hline & & & & & & & & & 1 & 1 \\
\hline \multirow[t]{4}{*}{1} & & & 1 & & & & & 2 & & 10 \\
\hline & & & & & & & & & & 2 \\
\hline & & 1 & & 1 & & & & & & 9 \\
\hline & & & & & & 1 & & & & 1 \\
\hline 5 & & 1 & & 1 & & 1 & & & & 11 \\
\hline 257 & 32 & 86 & 16 & 235 & 18 & 73 & 17 & 117 & 8 & 1393 \\
\hline
\end{tabular}


Tabla 2. Familias y número de especímenes de Hymenoptera muestreados por estratos de abril a octubre de 2012: (*) familias capturadas exclusivamente en el sotobosque; (**) familias capturadas exclusivamente en dosel

\begin{tabular}{|c|c|c|c|c|c|}
\hline \multicolumn{2}{|c|}{ HYMENOPTERA } & \multicolumn{2}{|c|}{ IV } & \multicolumn{2}{|c|}{ V } \\
\hline Superfamilia & Familia & dosel & sotob & dosel & sotob \\
\hline TENTHREDINOIDEA & Tenthredinidae & & 1 & 1 & \\
\hline \multirow[t]{2}{*}{ ICHNEUMONOIDEA } & Braconidae & 4 & & 19 & 9 \\
\hline & Ichneumonidae & 1 & & & 2 \\
\hline \multirow[t]{2}{*}{ EVANIOIDEA } & Evaniidae & & & & \\
\hline & Gasteruptionidae* & & & & \\
\hline \multirow[t]{2}{*}{ CERAPHRONOIDEA } & Megaspilidae & & 1 & & \\
\hline & Ceraphronidae* & & & & \\
\hline \multirow[t]{2}{*}{ PLATYGASTEROIDEA } & Scelionidae & 3 & 1 & 9 & 1 \\
\hline & Platygastridae & 9 & 2 & 60 & \\
\hline PROCTOTRUPOIDEA & Proctotrupidae* & & 1 & & \\
\hline \multirow[t]{10}{*}{ CHALCIDOIDEA } & Eulophidae & 1 & & 19 & 2 \\
\hline & Encyrtidae & & & 14 & 3 \\
\hline & Aphelinidae & 2 & 1 & 40 & \\
\hline & Eupelmidae & & & 1 & \\
\hline & Pteromalidae & 4 & 1 & 54 & 3 \\
\hline & Mymaridae & 1 & 1 & 12 & 10 \\
\hline & Eurytomidae** & & & & \\
\hline & Trichogrammatidae $* *$ & & & 2 & \\
\hline & Chalcididae** & & & & \\
\hline & Ormyridae** & & & & \\
\hline \multirow[t]{2}{*}{ CYNIPOIDEA } & Figitidae (Charipinae)** & & & 3 & \\
\hline & Cynipidae & 3 & 2 & 44 & 1 \\
\hline \multirow[t]{4}{*}{ CHRYSIDOIDEA } & Chrysidae & & & & \\
\hline & Bethylidae** & 1 & & 1 & \\
\hline & Dryinidae & & & & \\
\hline & Sclerogibbidae** & & & & \\
\hline \multirow[t]{3}{*}{ VESPOIDEA } & Pompilidae* & & & & \\
\hline & Mutillidae* & & & & \\
\hline & Formicidae & & 3 & 1 & 26 \\
\hline \multirow[t]{2}{*}{ APOIDEA } & Sphecidae & & & 1 & \\
\hline & Apidae** & & & 2 & \\
\hline TOTAL & & 29 & 14 & 283 & 57 \\
\hline
\end{tabular}




\begin{tabular}{|c|c|c|c|c|c|c|c|c|c|c|}
\hline \multicolumn{2}{|c|}{ VI } & \multicolumn{2}{|c|}{ VII } & \multicolumn{2}{|c|}{ VIII } & \multicolumn{2}{|c|}{ IX } & \multicolumn{2}{|c|}{$\mathbf{X}$} & \multirow[b]{2}{*}{ TOTAI } \\
\hline dosel & sotob & dosel & sotob & dosel & sotob & dosel & sotob & dosel & sotob & \\
\hline & & & & & & & & & & 2 \\
\hline \multirow[t]{4}{*}{22} & 22 & 18 & 3 & & 3 & 6 & & 5 & & 111 \\
\hline & & & & & & 1 & & & & 4 \\
\hline & & 2 & & & & & 1 & & & 3 \\
\hline & 1 & & & & & & & & & 1 \\
\hline \multirow[t]{2}{*}{5} & & & & & & 1 & & & & 7 \\
\hline & 4 & & & & & & & & & 4 \\
\hline 30 & 34 & 8 & 1 & & 1 & 3 & 1 & 3 & 3 & 98 \\
\hline \multirow[t]{2}{*}{17} & 3 & & & & & & & & 1 & 92 \\
\hline & & & & & & & & & & 1 \\
\hline 20 & 6 & & 1 & & & 2 & 1 & 1 & 1 & 54 \\
\hline 29 & 11 & 9 & 2 & & 4 & 2 & & 1 & & 75 \\
\hline 60 & 6 & 3 & 2 & 1 & & 1 & & & & 116 \\
\hline 2 & 7 & 2 & & & 1 & 1 & & & & 14 \\
\hline 13 & 5 & 6 & & & & & & 3 & & 89 \\
\hline \multirow[t]{3}{*}{24} & 26 & 10 & 6 & 1 & & 2 & & 2 & & 95 \\
\hline & & 1 & & & & & & & & 1 \\
\hline & & 2 & & & & & & & & 4 \\
\hline 1 & & & & & & & & & & 1 \\
\hline 1 & & & & & & & & & & 1 \\
\hline 3 & & & & & & & & & & 6 \\
\hline 14 & 8 & 1 & & 1 & & & & & & 74 \\
\hline \multirow[t]{3}{*}{5} & 2 & 2 & & & & & & & & 9 \\
\hline & & & & & & & & 1 & & 3 \\
\hline & 1 & 1 & & & & & & & & 2 \\
\hline \multirow[t]{3}{*}{1} & & & & & & & & & & 1 \\
\hline & 1 & & & & & & & & & 1 \\
\hline & 1 & & & & & & & & & 1 \\
\hline \multirow[t]{2}{*}{11} & 15 & 63 & 1 & 12 & 4 & 8 & 2 & 4 & 1 & 151 \\
\hline & 3 & 3 & & & & 1 & & & & 8 \\
\hline 8 & & & & & & & & & & 10 \\
\hline 266 & 156 & 131 & 16 & 15 & 13 & 28 & 5 & 20 & 6 & 1039 \\
\hline
\end{tabular}


De entre las familias capturadas exclusivamente en el dosel destaca Ceratopogonidae, cuyo aporte al total del volumen de Diptera en este estrato fue del 10,1\%. Cabe destacar además la actividad de la familia Bibionidae (Fig. 5), restringida al mes de abril y casi exclusivamente al dosel (96,5\%), aportando el 51\% del total muestreado en ese estrato durante ese mes. Igualmente, la familia Chloropidae destaca por haber desarrollado su actividad casi exclusivamente en el dosel $(98,7 \%)$. Durante el mes de junio, esta última familia aportó al conjunto de Diptera muestreado en el dosel el 62,8\% (Fig. 5). Solo el 12\% de los dípteros fueron capturados en el sotobosque durante el período estudiado (Tabla 1, Fig. 6), y gran parte de este material se debe al aporte de Cecidomyiidae (69\%).

Al igual que Diptera, Hymenoptera resultó uno de los grupos más abundantes en el área, representando el 13,3\% (1039 especímenes) del total de artrópodos muestreados durante el período de estudio. Un total de 31 familias de Hymenoptera fueron capturadas (Tabla 2), siendo las más abundantes Formicidae, con 151 especímenes para un 14,5\% del total de Hymenoptera (Fig. 7), Aphelinidae $(116 / 11,2 \%)$, Braconidae $(111 / 10,7 \%)$, Scelionidae $(98 / 9,4 \%)$, Mymaridae $(95 / 9,1 \%)$ y Platygastridae $(92 / 8,9 \%)$. En su conjunto, estas seis familias aportan el 63,8\% (663 especímenes) del total. Agosto resultó el mes con menor diversidad, con solo 8 familias capturadas, mientras junio ofreció el máximo, con 24 familias. En cuanto a la actividad, el máximo de todo el período se alcanzó en junio, con 422 especímenes, aportando Aphelinidae y Scelionidae el 30,8\% del total muestreado. La menor actividad se registró en octubre, con 26 especímenes.

Referente a la actividad de Hymenoptera registrada por estratos (Fig. 8), el dosel aportó el 74,3\% (772 especímenes) del total capturado en todo el período. De las 31 familias identificadas en total, 26 fueron capturadas en el dosel y 23 en el sotobosque, aunque, de estas, ocho familias se encontraron exclusivamente en el dosel mientras solo cinco lo fueron exclusivamente en el sotobosque (Tabla 2). De las seis familias más abundantes (Fig. 7), Platygastridae mostró su máxima actividad en mayo; Aphelinidae y Scelionidae, en junio, mientras que Formicidae alcanzaba su máximo en julio, desarrollando la casi totalidad de su actividad en el dosel durante ese mes. Braconidae, en cambio, desarrolló su actividad entre mayo y julio, con preferencia por el dosel $(63,4 \%)$. Es destacable que todas estas familias desarrollaron su actividad con preferencia en el dosel (Platygastridae, 93,5\%; Aphelinidae, 92,2\%; Braconidae, 66,7\%; Formicidae, 65,6\%; Scelionidae, 57,1\%; Mymaridae, 54,7\%).

\section{Discusión}

En general, la mayor parte de la actividad de la artropofauna en el bosque mediterráneo mixto se observó en el dosel (Fig. 3), donde se capturó el 70,6\% del total. En referencia a los Diptera, los resultados posicionan al dosel como el estrato donde se desarrolla el mayor volumen de actividad, atendiendo al total de especímenes capturados (Fig. 6) y a la mayor diversidad de familias registradas, 27 familias en contraposición a las 18 capturadas en el sotobosque. En el caso de los himenópteros, aunque no mostraron una diversidad significativamente superior en 

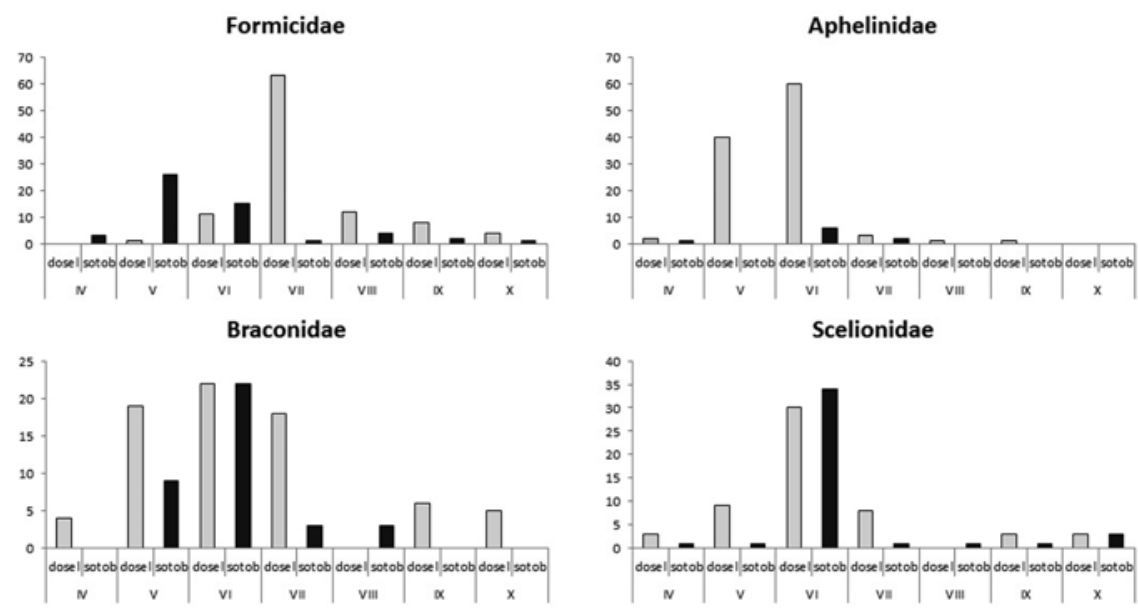

Mymaridae

Platygastridae
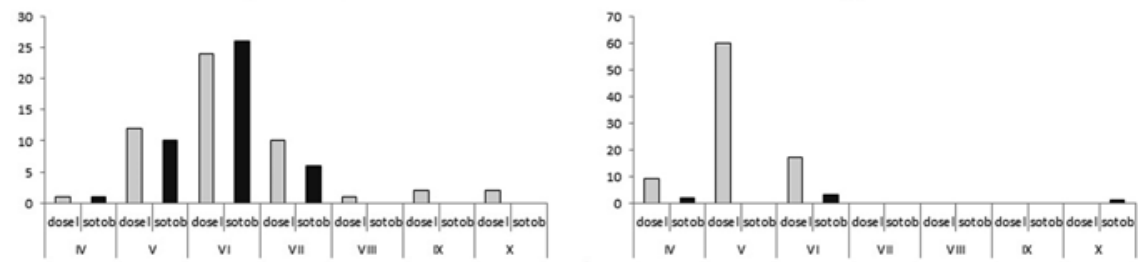

Figura 7. Actividad por estratos de las familias dominantes de Hymenoptera, de abril a octubre de 2012.

el dosel, ocho familias muestreadas exclusivamente en este estrato respecto a las cinco del sotobosque (Tabla 2), su abundancia resultó mayor en el dosel (Fig. 8). Además de estos dos órdenes mencionados, otros grupos de artrópodos voladores dominantes en el área (Hemiptera y Lepidoptera) desarrollaron también su mayor actividad en el dosel.
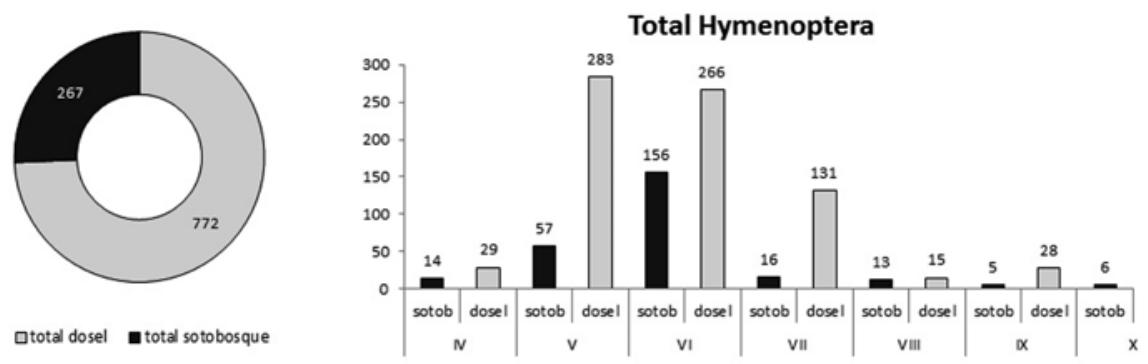

Figura 8. Total de especímenes de Hymenoptera capturados por estratos durante el estudio, de abril a octubre de 2012, y aporte por estrato y mes. 
Durante un estudio previo con trampa Malaise ubicada en el dosel de esta misma área, la captura de artrópodos en este estrato fue escasa, con un aporte muy pobre al conjunto de la fauna total muestreada entre los dos estratos (MederosLópez et al., 2012). Aunque podría establecerse como cierta la conjetura de que la ubicación de la Malaise durante este primer estudio influyó directamente en los resultados del mismo (trampa ubicada sobre el estrato o dosel conformado por Quercus ilex y $Q$. cerrioides pero por debajo del último estrato conformado únicamente por copas de Pinus halepensis Miller), este resultado debería esclarecerse con nuevos datos comparativos en un futuro. Se han identificado muchos factores primarios que influyen en la estratificación vertical de los artrópodos, como son el tiempo, la estructura forestal, la composición de la comunidad vegetal, los gradientes climáticos, la disponibilidad de recursos entre otros (Ulyshen, 2011). En nuestro caso, las observaciones de campo parecen corroborar una mayor actividad de la artropofauna en el estrato $Q$. ilex-Q. cerrioides, con una insolación completa y sin la presencia de un estrato superior de $P$. halepensis, lo que sugiere el efecto del gradiente climático como la causa principal de las diferencias tan acusadas en los resultados de las dos campañas.

Referente a la fenología de las familias dominantes de Diptera, Cecidomyiidae alcanzó su máximo en mayo, disminuyendo progresivamente su actividad hasta un nuevo pico en agosto (Fig. 5). En campañas anteriores se habían registrado similitudes en cuanto al máximo de actividad de esta familia (mayo y junio), pero con diferencias muy acusadas en cuanto a la actividad por estratos. Si en 20092010 la actividad máxima de Cecidomyiidae se registró en el sotobosque (95,5\%), en 2012 el 84,3\% de la actividad se registró en el dosel. La causa de esto podría encontrarse en la discusión anterior sobre la ubicación en el dosel de la trampa Malaise del estudio de 2009-2010. Por su parte, Chironomidae muestra un resultado similar a Cecidomyiidae en ambas campañas en lo que respecta a la actividad por estratos, con un $75,5 \%$ de la actividad registrada en el sotobosque durante 2009-2010 y 96\% en el dosel en 2012. Por otra parte, la actividad de Chironomidae parece estar correlacionada significativamente con las precipitaciones registradas en el área (Fig. 3 y 5), una observación ya realizada en 2009-2010. Aunque los 124 especímenes capturados de Ceratopogonidae lo ubican como una de las tres familias más numerosas, el 69,4\% de toda la actividad se desarrolló durante su pico máximo en agosto y exclusivamente en el dosel, siendo una familia más bien escasa el resto del período estudiado. Esta misma familia mostró una fenología muy diferente durante la campaña 2009-2010 (Mederos-López \& Pujade-Villar, 2011), en la cual el pico máximo se alcanzó en abril, manteniendo una actividad casi vestigial el resto del año, y el sotobosque aportó entonces el 86,5\% del total de especímenes muestreados.

Hemos podido confirmar la presencia casi exclusiva en el dosel de Zabrachia minutissima (Zetterstedt, 1838), la única especie de la familia Stratiomyidae observada en el área y muestreada en campañas anteriores en este estrato (mayoseptiembre) y con solo un ejemplar capturado en el sotobosque en la presente campaña (Tabla 1). Las larvas de esta especie, la única del género en la península Ibérica, se han encontrado viviendo bajo la corteza en descomposición de Pinus 
sylvestris (Boumans, 2005), en un bosque mixto con plantaciones de pino, abeto y roble. Dado que esta especie de pino no se encuentra en nuestra zona de estudio, podría especularse sobre la posible utilización de Pinus halepensis por parte de $Z$. minutissima como especie arbórea donde completar su ciclo biológico. Estos datos sugieren que el ciclo de vida de Z. minutissima incluye un cambio entre los estratos del bosque, desarrollándose su larva bajo la corteza de troncos en descomposición, cerca del suelo, y pasando al dosel cuando emergen los adultos para alimentarse y reproducirse.

Otro ejemplo de la fauna de Diptera encontrada exclusivamente en el dosel es la especie Cryptochetum buccatum Hendel, 1933 (Cryptochetidae), con cuatro especímenes muestreados de julio a septiembre (Tabla 1). Carles-Tolrá (1993) la cita de Barcelona, definiéndola entonces como especie rara. En este sentido, especies que se han considerado raras durante mucho tiempo han resultado ser frecuentes cuando se han comenzado a dirigir estudios focalizados en el dosel. Stark (2008) determinó que el género Oedalea (Hybotidae), cuyos adultos son poco frecuentes entre el material muestreado mediante métodos convencionales, formaba parte de la fauna característica del dosel de los bosque templados de Europa tras estudiar un abundante material como resultado de la fumigación (fogging) de este estrato. De igual forma, un buen número de nuevas especies de diversas familias de Diptera y otros grupos, así como de nuevos registros para la fauna europea, han sido descubiertas a partir de estudios focalizados en el dosel (Bouget et al., 2011; Diestelhorst \& Lunau, 2001; Thunes et al., 2004, 2008; Papp \& Fölvary, 2001).

Además de estos ejemplos, dos de las diez familias más abundantes muestreadas en el área se capturaron exclusivamente en el dosel, Pipunculidae y Tachinidae (Tabla 1). Igualmente, destaca también la familia Chloropidae, al muestrearse en casi el $98 \%$ en el dosel durante el período estudiado. Otros estudios han revelado especímenes de Chloropidae en gran abundancia en el dosel, utilizando otros métodos de muestreo diferentes al empleado por nosotros (Lepointe, 1956).

En relación con la fauna de Hymenoptera, los datos fenológicos obtenidos anteriormente para algunas de las familias en esta misma área y durante los mismos meses (Mederos-López et al., 2012) no son coincidentes en muchos casos con los obtenidos en el presente estudio. Como se ha explicado anteriormente en lo referente a la trampa situada en el dosel dominado por $Q$. ilex-Q. cerrioides con insolación completa, en el estudio anterior se encontraba bajo la influencia de un estrato superior conformado exclusivamente por $P$. halepensis, situación que no se da en este estudio. Esta distinta ubicación podría explicar en parte las diferencias en los resultados; no obstante, un análisis más minucioso es necesario para cotejar distintas variables. Una situación muy diferente es la observada en los resultados del sotobosque; aunque el número de ejemplares muestreados es distinto, prácticamente se han colectado las mismas familias en ambos estudios.

Un aspecto a destacar de la fauna de Hymenoptera es la ausencia durante esta campaña de la familia Diapriidae, dominante durante el estudio previo realizado en 2009-2010 (Mederos-López et al., 2012). Con 2222 especímenes, Diapriidae representaba entonces el $33 \%$ del total de Hymenoptera, un aporte muy significa- 
tivo al conjunto de la fauna. Por lo general, son parasitoides en las larvas y pupas de una amplia gama de insectos, especialmente moscas, y algunas especies son hiperparasitoides (por ejemplo de Cicadellidae). Posiblemente, la presencia/ausencia de los huéspedes podría explicar este evento de emergencia. Son bien conocidas las explosiones de fauna parasitoide cuando sus huéspedes son muy abundantes, pero en ausencia de estos los parasitoides faltan. Se necesitaría determinar a nivel específico los especímenes muestreados durante la campaña 20092010 para intentar encontrar una explicación a la ausencia total de esta familia durante la presente anualidad.

También es interesante señalar que la abundancia de capturas en el dosel ha sido siempre superior a la del sotobosque en prácticamente todos los grupos colectados y durante todos los meses estudiados (Tabla 2). Este hecho, sumado a la presencia de una mayor diversidad de familias en el dosel, ubica a este último como el estrato preferente para los himenópteros donde se desarrolla el grueso de la actividad del grupo, a pesar de lo que podría esperarse en un principio. En este sentido, coincidimos con los resultados de Sobek et al. (2009) durante un estudio sobre la abundancia y riqueza de especies de Hymenoptera en bosques templados de Europa, en cuanto a la existencia de una estratificación espacial distinta en que la abundancia de abejas, avispas y parasitoides fue superior en el dosel que en el sotobosque. Por otro lado, cabe destacar que muchas de las familias con especímenes muy pequeños, como Encyrtidae, Aphelinidae, Mymaridae, Trichogrammatidae, Scelionidae, entre otros, han sido colectadas en mayor medida en el dosel, cuando los huéspedes habituales de estos grupos se encuentran sobre todo en el sotobosque. Ello podría deberse a una estrategia de dispersión en la que el viento, marcadamente más intenso en el dosel, jugara un papel esencial, por lo que las especies de estas familias subirían a las partes más altas del bosque para ser desplazadas pasivamente antes de encontrar a los huéspedes.

En lo que se refiere a familias concretas, podemos comentar que en el caso de la familia Cynipidae tan solo hemos colectado especímenes del género Plagiotrochus, observado anteriormente en el área y en las mismas mensualidades (Mederos et al., 2012), pero con un número de ejemplares mucho mayor (1:74). La distinta ubicación de la trampa y la posibilidad de ser desplazados por el viento podrían ser las causas. Este proceso de transporte ya fue comentado para la especie Plagiotrochus amenti Kieffer, 1901 en Ros-Farré \& Pujade-Villar (1998). También debemos destacar que en los Figitidae tan solo ha sido capturada la subfamilia Charipinae, mientras que en Mederos et al. (2012) se muestreó la subfamilia Eucoilinae. Finalmente, es interesante señalar que hay familias que solo han sido capturas o bien en el dosel o bien en el sotobosque (Tabla 2), lo cual está relacionado con aspectos anteriormente comentados o a la peculiar biología de cada familia y especie; así por ejemplo, Pompilidae, Mutillidae y Gasteruptionidae no han sido muestreadas en el dosel, lo que concuerda con los hábitos y ecología de las especies de estas familias, ya que tienen sus huéspedes a ras de suelo.

En conclusión, podemos afirmar que el dosel del bosque mixto mediterráneo en el área estudiada ofrece recursos suficientes como para albergar una amplia 
diversidad de artrópodos, en particular Diptera e Hymenoptera, existiendo incluso ciertos taxones que de momento se han encontrado restringidos a este estrato. Hemos encontrado una clara estratificación de la fauna, y el dosel se muestra no solo como el estrato donde se desarrolla el mayor volumen de la actividad de los artrópodos, sino también como el estrato más biodiverso a juzgar por el número de grupos encontrados en él en contraposición a los capturados en el sotobosque. Esto no excluye que muchas de las especies muestreadas en fase adulta en el dosel, algunas incluso encontradas exclusivamente en este estrato, pasen una parte de su desarrollo biológico cerca del suelo, sobre todo en los primeros estadios, migrando al dosel posteriormente tras la emergencia. Las marcadas diferencias entre los resultados del primer estudio durante la campaña 2009-2010 y la descrita en este trabajo, evidencian la necesidad de estudiar los diversos factores que marcan la actividad de los artrópodos en un estrato de dosel $Q$. ilex-Q. cerrioides con insolación total respecto a uno con presencia de un estrato superior conformado por $P$. halepensis. Aunque ciertas observaciones apuntan a la temperatura y la disponibilidad de la luz solar, la influencia de otros factores como la exposición al viento, las fluctuaciones de la humedad relativa, entre otros, podrían esclarecer las diferencias en los resultados de ambas campañas. Por último, la rica diversidad encontrada en el dosel de Collserola expone la necesidad de incluir este hábitat y los recursos que ofrece dentro de los planes de manejo forestal que realiza el órgano gestor del parque natural, en aras de mantener la diversidad de estas comunidades forestales de artrópodos.

\section{Agradecimientos}

A Lluís Cabañeros y resto del personal del Consorci del Parc Natural de la Serra de Collserola y de la Estació Biològica del parque, así como a Rafel Cebrian, del Parc Zoològic de Barcelona, por el apoyo y las facilidades ofrecidas durante todas las etapas del estudio. A los mecenas del proyecto durante su participación en \#SciFund Challenge, y en especial a Nalini Nadkarni, Iñaki Gorostidi, Mary Canady, Jai Ranganathan y Jarrett Byrnes. Este estudio se ha realizado gracias al apoyo del Consorci del Parc Natural de la Serra de Collserola y a las ayudas y subvenciones del Parc Zoològic de Barcelona y del Ajuntament de Barcelona, correspondientes al período 2012.

\section{Referencias bibliográficas}

Bentanachs, J.; Viñolas, A.; Mederos, J. 2012. Primer registro de Longitarsus substriatus Kutschera, 1862 para la Península Ibérica (Coleoptera: Chrysomelidae: Alticinae). Elytron 25: 111-115.

Bouget, C.; Brin, A.; Brustel, H. 2011. Exploring the «last biotic frontier»: Are temperate forest canopies special for saproxylic beetles? Forest Ecology and Management 261 (2): 211-220.

Boumans, L. 2005. Zabrachia minutissima found in The Netherlands after all (Diptera: Stratiomyidae). Entomologische Berichten 65 (4): 132-134. 
Carles-Tolrá, M. 1993. Algunas especies de dípteros nuevas o interesantes para España peninsular (Díptera, Acalyptratae). Boletín de la Asociación Española de Entomología 17 (1): 9-18.

Diestelhorst, O.; Lunau, K. 2001. Leben in der Kronen. Farbschalenfänge von Dolichopodiden im Kronenraum einer Buche. Mitteilungen der Deutschen Gesellschaft für allgemeine und angewandte Entomologie 13: 543-546.

Lepointe, J. 1956. Méthodes de capture dans l'écologie des arbres. Vie et Milieu 7 (2): 233-241.

Mederos-López, J.L.; Pujade-Villar, J. 2011. Actividad nictemeral y anual de los Diptera (Insecta) en un bosque mediterráneo mixto de Cataluña. Orsis 25: 83-103.

Mederos-López, J.; Mata-Casanova, N.; Pujade-Villar, J. 2012. Fenología anual y actividad nictemeral de los Hymenoptera (Insecta) en un bosque mixto mediterráneo. Orsis 26: 201-225.

Mederos-López, J.; Baz-Ramos, A. 2012. Psocoptera de un bosque mixto mediterráneo del Parc Natural de la Serra de Collserola (Cataluña, España) Orsis 26: 227-233.

Mitchell, A. W.; Secoy, K.; Jackson, T. (Eds.). 2002. The Global Canopy Handbook: Techniques of Access and Study in the Forest Roof. Global Canopy Programme. Oxford.

Moffett, M.; Lowman, M. 1995. Canopy access techniques. In: Lowman, M. \& Nadkarni, N. (Eds.). Forest Canopies, San Diego. p. 3-26.

Oosterbroek, P. 2006. The European Families of the Diptera. Identification, diagnosis, biology. KNNV Publishing. Utrecht.

Papp, L.; Fölvary, M. 2001. A new genus and three new species of Hybotidae with new records of the Hungarian Empidoidea (Diptera). Acta Zoologica Academiae Scientiarum Hungaricae 47 (4): 349-361.

Pujade-Villar, J.; Fernández-Gayubo, S. 2004. Himenópteros. In: Barrientos, J.A. (Ed.). Curso práctico de entomología. Manuals Universitat Autònoma de Barcelona, 41: 813854.

Ros-Farré, P. \& Pujade-Villar, J. 1998. Estudio mediante una trampa Malaise de la comunidad de cinípidos cecidógenos e inquilinos de Santa Coloma, Andorra (Hymenoptera, Cynipidae). Ecología, 12: 441-454.

Sobek, S.; Tscharntke, T.; Scherber, C.; Schiele, S.; Steffan-Dewenter, I. 2009. Canopy vs. understory: Does tree diversity affect bee and wasp communities and their natural enemies across forest strata? Forest Ecology and Management 258 (5): 609-615.

Stark, A. 2008. Species of the genus Oedalea Meigen, 1820 (Diptera: Hybotidae): An element of the canopy fauna in European forests?. In: Floren, A. \& Schmidl, J. (Eds.). Canopy arthropod research in Europe. bioform entomology. Nuremberg. p. $105-117$.

Thunes, K.H.; Skartveit, J.; Gjerde, I.; Starý, J.; Solhøy, T.; Fjellberg, A.; Kobro, S.; Nakahara, S.; zur Strassen, R.; Vierbergen, G.; Szadziewski, R.; Hagan, D.V.; Grogan, W.L.; Jonassen, T.; Aakra, K.; Anonby, J.; Greve, L.; Aukema, B.; Heller, K.; Michelsen, V.; Haenni, J.-P.; Emeljanov, A.F.; Douwes, P.; Berggren, K.; Franzen, J.; Disney, R.H.L.; Prescher, S.; Johanson, K.A.; Mamaev, B.; Podenas, S.; Andersen, S.; Gaimari, S.D.; Nartshuk, E.; Søli, G.E.E.; Papp, L.; Midtgaard, F.; Andersen, A.; Tschirnhaus, M.; Bächli, G.; Olsen, K.M.; Olsvik, H.; Földvári, M.; Raastad, J.E.; Hansen, L. O.; Djursvoll, P. (2004). The arthropod community of Scots pine (Pinus silvestris L.) canopies in Norway. Entomologica Fennica 15: 65-90.

Thunes, K.H.; Gjerde, I.; Hagan, D.V.; Szadziewski, R. 2008. Search the canopies and you will find new species of insects. In: Floren, A. \& Schmidl, J. (Eds.). Canopy arthropod research in Europe. bioform entomology. Nuremberg. p. 215-223. 
Ulyshen, M.D. 2011. Arthropod vertical stratification in temperate deciduous forest: Implications for conservation-oriented management. Forest Ecology and Management 261: 1479-1489.

Viñolas, A. 2011a. Nuevas localizaciones de anóbidos para la Península Ibérica (Coleoptera: Bostrichoidea). Elytron 24: 3-18.

Viñolas, A. 2011b. Noves aportacions a la distribució del gènere Caenocara Thomson, 1859, Dorcatominae, a Catalunya (Coleoptera: Bostrichoidea). Orsis 25 (2010): 121130

Viñolas, A.; Soler, J.; Muñoz Batet, J. 2012a. Nuevos registros y nuevas localizaciones de coleópteros para la Península Ibérica y en especial del Paratge Natural de l'Albera, Girona (Coleoptera). Elytron 25: 3-63.

Viñolas, A.; Muñoz, J.; Soler, J. 2012b. Noves o interessants citacions de coleòpters per al Parc Natural del Montseny i per a la Península Ibèrica (Coleoptera) (4a nota). Orsis 26: 149-185.

Viñolas, A. 2013a. Els Mesocoelopodinae de la península Ibèrica i illes Balears. Els gèneres Mesocoelopus Jacquelin du Val, 1860, Mesothes Mulsant \& Rey, 1864 i Rhamna Peyerimhoff, 1913 (Coleoptera: Ptinidae). Butlletí de la Institució Catalana d'Història Natural 76: 105-116.

Viñolas, A. 2013b. Els Dorcatominae de la península Ibèrica i illes Balears. 1a nota. Els gèneres Caenocara C.G. Thomson, 1859, Calymmaderus Solier, 1849 i Mizodorcatoma Hayashi, 1955 (Coleoptera: Ptinidae). Butlletí de la Institució Catalana d'Història Natural 76: 117-132.

Viñolas, A. 2013c. Els Dorcatominae de la península Ibèrica i illes Balears. 2a nota. El gènere Dorcatoma Herbst, 1792 (Coleoptera: Ptinidae). Orsis 27: 7-28.

Viñolas, A. 2013d. Els Dorcatominae de la península Ibèrica i illes Balears. 3a nota. El gènere Stagetus Wollaston, 1861, amb la descripció de S. confusus n. sp. (Coleoptera: Ptinidae). Orsis 27: 95-121.

Viñolas, A.; Masó, G. 2013. Sobre la presencia de Clambus dux dux Endrödy-Younga, 1960 en la Península Ibérica y revisión preliminar de los Clambidae ibéricos (Coleoptera). Arquivos Entomoloxicos 9: 59-72. 
